\title{
Feature selection via dimensionality reduction for object class recognition
}

\begin{abstract}
This paper investigates the effects of feature selection via dimensionality reduction techniques for the task of object class recognition. Two filter-based algorithms are considered namely Correlation-based Feature Selection (CFS) and Principal Components Analysis (PCA). A Support Vector Machine is used to compare these two techniques against classical feature concatenation, based on the Graz02 dataset. Experimental results show that the feature selection algorithms are able to retain the most relevant and discriminant features, while maintaining recognition accuracy and improving model building time.
\end{abstract}

Keyword: Feature fusion; Feature selection; Filter model; Object class recognition; Support vector machine 